\title{
Anisotropy of elastic deformations in multilayer (In,Ga)As/GaAs structures with quantum wires: $X$-ray diffractometry study
}

\author{
V.V. Strelchuk ${ }^{1}$, V.P. Kladko ${ }^{1}$, O.M. Yefanov ${ }^{1}$, O.F. Kolomys ${ }^{1}$, O.I. Gudymenko ${ }^{1}$, M.Ya. Valakh ${ }^{1}$, \\ Yu.I. Mazur ${ }^{2}$, Z.M. Wang ${ }^{2}$, and G.J. Salamo ${ }^{2}$ \\ ${ }^{1}$ V. Lashkaryov Institute of Semiconductor Physics, NAS of Ukraine, 45, prospect Nauky, 03028 Kyiv, Ukraine \\ E-mail: strelch@isp.kiev.ua \\ ${ }^{2}$ University of Arkansas, Department of Physics, Fayetteville, 72701 Arkansas
}

\begin{abstract}
Using the method of high-resolution X-ray diffraction (HRXRD), we have studied 17-period $\mathrm{In}_{0.3} \mathrm{Ga}_{0.7} \mathrm{As} / \mathrm{GaAs}$ multilayer structure with self-assembled quantum wires (QWRs) grown by the MBE and subjected to postgrowth rapid thermal annealing (RTA) at temperatures $\left(T_{\text {ann }}\right)$ from 550 to $850{ }^{\circ} \mathrm{C}$ for $30 \mathrm{~s}$. It has been shown that the spatial arrangement of QWRs (lateral and vertical) causes the quasi-periodical strain distribution, the strains being essentially anisotropic relatively to crystallographic directions of $\langle 011\rangle$ type. At $T_{\text {ann }} \leq 750{ }^{\circ} \mathrm{C}$, the driving mechanism of structural transformations is relaxation of residual strains due to thermally-activated and strainenhanced processes of In/Ga atom interdiffusion at the interface QWRs-2D layer, which does not result in considerable changes of the In concentration in (In,Ga)As QWRs. The presence of two superlattice vertical periods in the samples under study and their changes during RTA we explained by an anisotropic character of elastic strain distribution and lowered structure symmetry. The revealed increase in the (In,Ga)As QWRs lateral period caused by RTA is a direct evidence of running lateral mass-transfer processes and can be explained using the model "nucleation plus strain-enhanced $\mathrm{In} / \mathrm{Ga}$ atom lateral interdiffusion". At low annealing temperatures, there takes place dissolution of intermediate QWRs as a result of interdiffusion enhanced by residual anisotropic strains. At high RTA temperatures, the interdiffusion process is mainly determined by the composition gradient existing between QWRs and 2D layer.
\end{abstract}

Keywords: multilayer structures, quantum wires, elastic straines, X-ray diffraction.

Manuscript received 14.01.05; accepted for publication 18.05.05.

\section{Introduction}

Unique electrophysical and optical properties of semiconductor nanostructures with quantum dots (QDs) and quantum wires (QWRs) obtained in Stranski Krastanow mode using epitaxy of strained heterosystems open new perspectives to create modern devices for nano- and optoelectronics, for instance, laser diodes with a low threshold current and power consumption [1]. From the viewpoint of practical applying of these nanostructures, it is very important to reduce QDs sizes, to increase the density of their surface distribution and the ordering. Multilayer structures with spatially ordered QDs and QWRs are characterized by high homogeneity of their sizes, shapes as well as distances between QDs and QWRs. As it was recently shown, growing the (In,Ga)As/GaAs multilayer structures provides obtaining of laterally ordered chains of (In,Ga)As QDs with the length up to $5 \mu \mathrm{m}$ [2] and (In,Ga)As QWRs [3, 4]. The process of thermal annealing enables one to improve homogeneity of QDs and QWRs size distribution. For example, as a result of the thermal annealing of singlelayer (In,Ga)As/GaAs QDs [5] there was improved QDs size homogeneity and was shown the possibility of gradual changing the emission wave length. It was found that strain-enhanced atom interdiffusion [6] plays an important role in the process of InAs QDs annealing. However, up to date, the mechanism of $\mathrm{In} / \mathrm{Ga}$ interdiffusion and structural transformations in (In,Ga)As QDs and, moreover, in structures with (In,Ga)As QWRs has not been ascertained yet. The investigation of structural and optical properties of spatially ordered multilayer structures as well as their changes in the course of the thermal annealing is of great interest to understand mechanisms of their epitaxial growth and formation, to study the features of elastic strain relaxation and generation of structural defects. 
a)

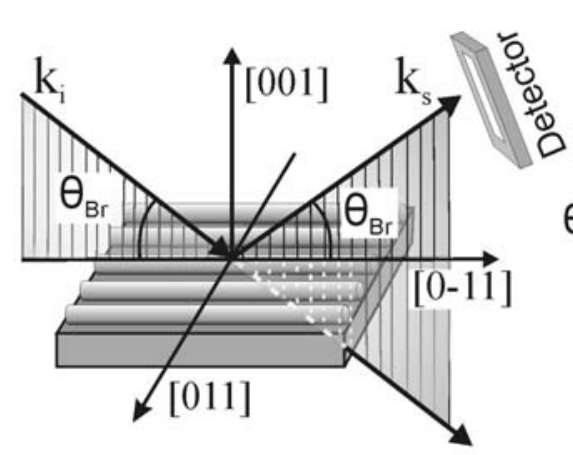

c)

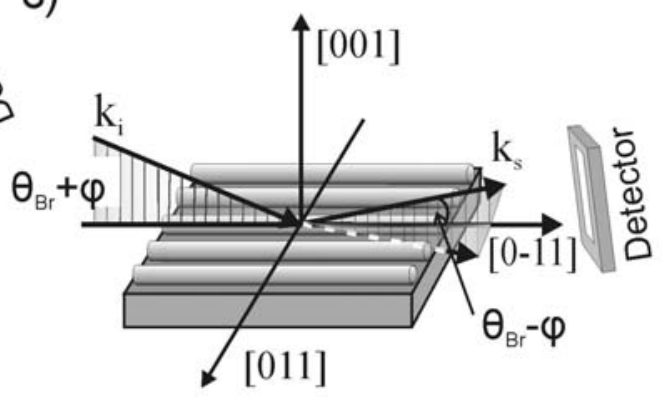

b)
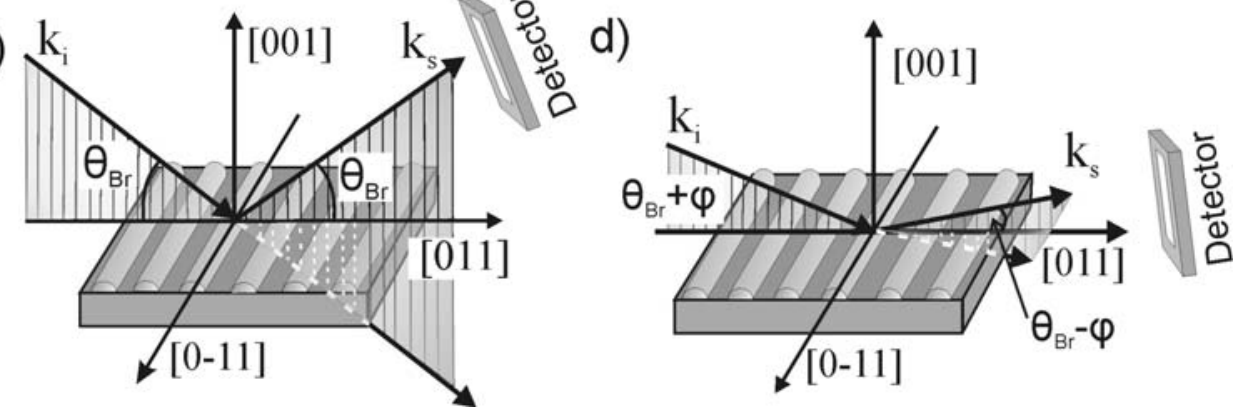

Fig. 1. Schematic representation of experimental geometry for studying quantum wires: $a, b$ - for 004 symmetrical reflection; c, d -311 asymmetrical reflection, where $\varphi$ is the angle between the reflection plane and surface.

It is known that 2D-3D transition and the process of three-dimensional QDs (or QWRs) formation in the course of epitaxial deposition of InGaAs/GaAs layers essentially changes the character of the elastic strain distribution as compared with that in planar layers. In multilayer structures, relaxation of elastic strains induced by QDs or QWRs [7] is realized through their spatial arrangement. In this case, there takes place an anisotropic character of the strain distribution relatively to crystallographic directions of $\langle 011\rangle$ type [8], which determines an anisotropy of structural parameters (for example, lowering the structural symmetry [9]) and was observed in planar heterostructures $[10,11]$. This structural anisotropy influences on X-ray diffraction, which allows to use high-resolution X-ray diffractometry in investigations of elastic strain anisotropy in nanostructures with QDs and QWRs.

In this work, adduced are the results of structural investigations of multilayer (In,Ga)As/GaAs (100) structures with self-assembled (In,Ga)As QWRs after RTA by HRXRD. The obtained results allows to ascertain regularities of structural changes in the course of the thermal annealing as well as to study peculiarities of elastic strain relaxation in the spatially ordered (In,Ga)As QWRs, which is of great importance for understanding physical properties of quantum-sized objects.

\section{Sample}

The samples used in this investigation were grown on semi-insulating GaAs (100) substrates with a miscut smaller than $0.05^{\circ}$ by conventional solid-source molecular beam epitaxy (MBE). After the native oxide was desorbed from the GaAs substrate surface at $650{ }^{\circ} \mathrm{C}$ for $10 \mathrm{~min}$, the GaAs buffer layer of $500 \mathrm{~nm}$ thickness was grown at $T=600{ }^{\circ} \mathrm{C}$ and the growth rate about one monolayer (ML) per second. After that, the substrate temperature was reduced down $540{ }^{\circ} \mathrm{C}$, and 17 periods of (11.5ML) $\mathrm{In}_{0.3} \mathrm{Ga}_{0.7} \mathrm{As} /(67 \mathrm{ML}) \mathrm{GaAs}$ were deposited. The growth rates of $\mathrm{GaAs}$ and $\mathrm{In}_{0.3} \mathrm{Ga}_{0.7} \mathrm{As}$ were equal to 0.4 and $0.8 \mathrm{ML} / \mathrm{s}$, respectively. $\mathrm{As}_{4}$ beam equivalent pressure was $10 \mu$ Torr for the whole structure growth. The growth interruptions were introduced during the deposition of the GaAs spacer [12].

Rapid thermal annealing was performed in argon atmosphere at temperatures 550 to $850^{\circ} \mathrm{C}$ for $30 \mathrm{~s}$. The time of setting the operation temperature was $10 \mathrm{~s}$.

The structural morphology of the sample surface was investigated using atomic force microscopy (AFM) in the tapping mode.

\section{Experimental details}

The X-ray scattering experiments were performed using double-crystal diffractometer $(\mathrm{GaAs}(100)$ crystalmonochromator, $\mathrm{Cu} K_{\alpha}$ radiation, the fourth reflection order) for symmetrical 400 and asymmetrical 311 reflections. The sample was scanned in the vicinity of the exact Bragg position close to $3^{\circ}$ using the so-called $\omega / 2 \theta$-mode. The measurements were performed in the discrete angular mode with the step $2 "$. The registration was realized in the accumulation regime for the pulse quantity measured at the signal-to-noise ratio $\leq 10^{-4}$. In 

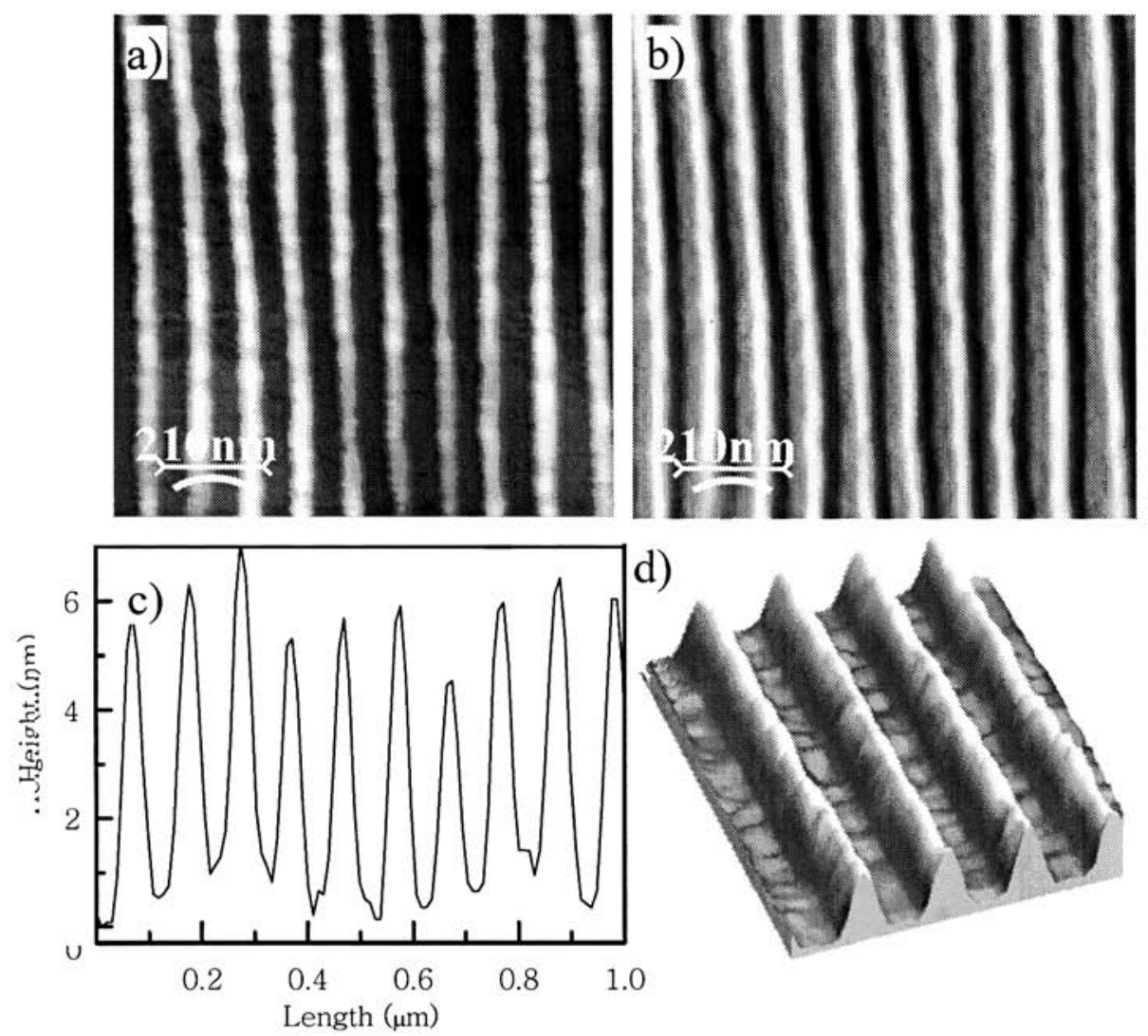

Fig. 2. AFM 1- $\mu \mathrm{m}$ images of the upper InGaAs layer of 17-period (In, Ga)As/GaAs multilayer with quantum wires (a) as well as $(d z / d x)$ derivative of AFM image (b). The cross-section of the surface profile (c) along the white line on the Fig. 2a and a fragment of 3D $0.5 \times 0.5 \mu \mathrm{m}$ AFM image of the structure with (In, Ga)As QWRs (d).

our analysis of the experimental data, we compared experimental and calculated rocking curves (RCs) by the way of fitting that uses the so-called $\chi^{2}$-method, which enabled us to obtain averaged parameter values and their accuracies.

$\mathrm{X}$-ray investigations were carried out both in symmetric and asymmetric Bragg reflection geometry when radiation was directed along two azimuthal crystallographic directions - parallel and perpendicular to the orientation of (In, Ga)As QWRs (Fig. 1).

RCs taken in the symmetric Bragg reflection geometry 004 (Fig. 1a, b)(the Bragg angle $\theta_{\mathrm{Br}}=33.02$, the penetration depth is of the order of $3 \mu \mathrm{m}$ ) allows to determine the only structural parameters in the direction perpendicular to the layer plane. While RCs, taken in the asymmetric reflection 311 using the grazing incidence $\mathrm{X}$ ray scattering (Fig. lc, d) when $\theta_{\mathrm{Br}}-\varphi=1.625$ relatively to the sample surface, are extremely sensitive to the parameters of the crystal structure in the layer plane.

The measured RC corresponded to a laterally averaged crystal structure of the multilayer sample (the width of the coherent X-ray beam at the sample surface is equal to several millimetres). When positioning the sample, we tried to provide the maximum intensity of the GaAs substrate reflection peak.

\section{Results and discussion}

Shown in Fig. 2 is the AFM image of the upper uncovered InGaAs layer of 17-period $\operatorname{In}_{0.3} \mathrm{Ga}_{0.7} \mathrm{As} / \mathrm{GaAs}$ multilayer with quantum wires. The surface morphology of the initial sample is an array of periodically located QWRs. The mean distance between wires is approximately $90 \mathrm{~nm}$, and the width of their base changes within the limits 35 to $41 \mathrm{~nm}$. It was ascertained that there takes place the bimodal distribution of QWRs heights in structures under investigation: their mean values are equal to 3.5 and $6.5 \mathrm{~nm}$. (In, Ga)As QWRs are oriented along [0-11] direction and possess the length larger than $5 \mu \mathrm{m}$.

Fig. 3 shows a RCs for 004 reflection of the initial and annealed samples in the case when the direction of incident radiation coincides with the crystallographic direction [011] that is perpendicular to the (In, Ga)As QWRs orientation direction (Fig. 1a): A (initial), B (annealed, $500^{\circ} \mathrm{C}$ ), C (annealed, $600^{\circ} \mathrm{C}$ ), D (annealed, $650^{\circ} \mathrm{C}$ ), $\mathrm{E}$ (annealed, $750^{\circ} \mathrm{C}$ ), and $\mathrm{F}\left(\right.$ annealed, $850^{\circ} \mathrm{C}$ ). $\mathrm{S}_{\mathrm{A}}$ and $-\mathrm{S}_{\mathrm{F}}^{r}$ curves correspond to simulated RCs for the samples $\mathrm{A}$ and $\mathrm{F}$, respectively. These RCs were modelled using the dynamical theory of X-ray diffraction and being based on the Takagi - Taupin model. It was assumed that $(\mathrm{In}, \mathrm{Ga}) \mathrm{As} / \mathrm{GaAs}$ is a multilayer structure 


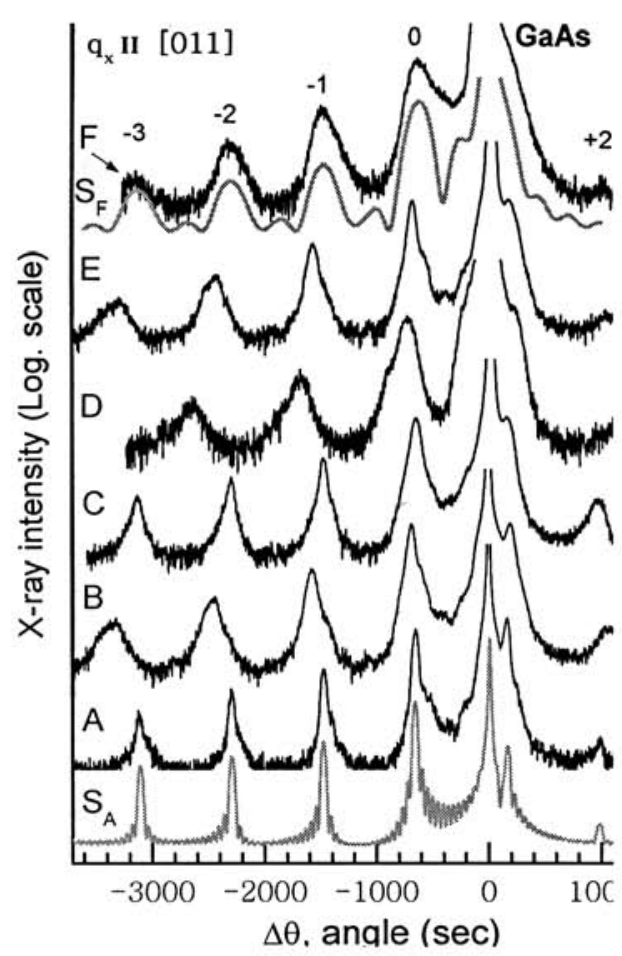

Fig. 3. Double-axis rocking curves for 17-period (In,Ga)As/GaAs multilayer with (In,Ga)As quantum wires (curves $\mathrm{A}-\mathrm{F}$ ) together with the simulated ones (curves $\mathrm{S}_{\mathrm{A}}$ and $\mathrm{S}_{\mathrm{F}}$ ). The curve A describes the initial sample, while curves B F correspond to the RTA temperatures $550,600,650,750$ and $850^{\circ} \mathrm{C}$, respectively. GaAs 004 reflection, $\mathrm{Cu} K_{\alpha 1}$ radiation. Curves are vertically shifted for convenience. Spectra are normalized by the intensity of zero satellite.

with (In,Ga)As QWRs similar to the tetragonal lattice distortion (In, Ga)As(wetting layer)/GaAs(barrier) multilayer structure with quantum wells. Technological parameters of these structures were used as initial conditions when fitting the theoretical RC spectra to the experimental ones. Final fitting to the parameter value $\chi=1.34$ was reached by introduction of various In concentration and layer thickness values into our calculations. As a result of this procedure, we have obtained the layer thickness in the superlattice period, In concentration and the lattice parameter in the direction of structure growth. This approach has already been used in $[13,14]$ when studying the multilayer InAs/GaAs structure with QDs, which enabled one to estimate In concentration in $2 \mathrm{D} \mathrm{InGaAs}$ wetting layer.

Although the RC of the multilayer structure in the case of 004 reflection is rather complex, there are several characteristic features usually used in its analysis. RC of the initial sample (Fig. 3, curve A) contains: I) GaAs substrate peak; II) zero peak or the peak of the mean mismatch caused by superposition of the Bragg reflections for two components of the multilayer quantum structure $\mathrm{GaAs}$ and InGaAs. Its angular position allows to estimate the average In concentration value $x_{\mathrm{av}}$ in the structure; iii) set of the secondary peaks - "satellites" symmetrically surrounding the above zero peak. Periodicity of these satellites is determined by the thickness of the layers that are repeated, that is corresponds to the period of the multilayer structure. By another words, the interferential oscillations corresponds to interlayer interference of the multilayer structure, which is an analogy of optical interference in thin films. All that is indicative of a high structure crystal perfectness, planarity of interfaces and very low density of extended defects that can be created during their epitaxial growing. It is worth to pay attention to some features of the curve A as compared to the simulated curve $\mathrm{S}_{\mathrm{A}}$ (Fig. 3). The shape of reflection peaks is asymmetric from the side of small angles, which can be explained by the presence of azimuthal anisotropy of the elastic strain distribution (in detail this effect will be considered below).

As seen from Fig. 3, in general the shape of RCs and the number of satellites (shown are 5 satellites) are similar to those of initial and annealed samples. This fact can indicate that RTA (for $30 \mathrm{~s}$ ) under all the used temperatures does not result in any dramatic changes in interface and structure quality of the samples with (In, Ga)As QWRs. However, with increasing the RTA temperature, except the shift of the satellite positions $\Delta \theta^{(i)}$ we could observe two effects: decrease in intensity of all the reflection peaks as compared to that of $\mathrm{GaAs}$ as well as asymmetric increase in the satellite halfwidth. The fact that the satellites can be reliably registered in the case of annealed samples and their intensity decreases a little as compared with that of the initial sample can be indicative of insignificant smoothing the interfaces in the result of interdiffusion processes at the heteroboundaries $\mathrm{GaAs}$ spacer layer - $2 \mathrm{D}$ InGaAs wetting layer.

Broadening the satellite peaks can be caused by several reasons:

1) dislocations that do not change the peak angular position but causes their broadening and appearance of the diffuse scattering. In our samples, the presence of dislocations is unlikely, which is confirmed by the results of electron microscopy investigations of the similar structures grown in the same technological regimes [15]. An indirect confirmation of the made conclusion is the change of satellite angular positions in annealed samples and in spectra obtained for two mutually orthogonal directions of incident radiation (Fig. 5);

2) roughness of interfaces and the gradient composition. These two characteristics cannot be distinguished when, analyzing the experimental two-axis RC. Theoretical calculations [16] show that the interface roughness results in broadening the superlattice satellite peaks in proportion to $n$, where $n$ is the satellite order. As seen in Fig. 3, this effect is not dominant in our case but can manifest itself in 


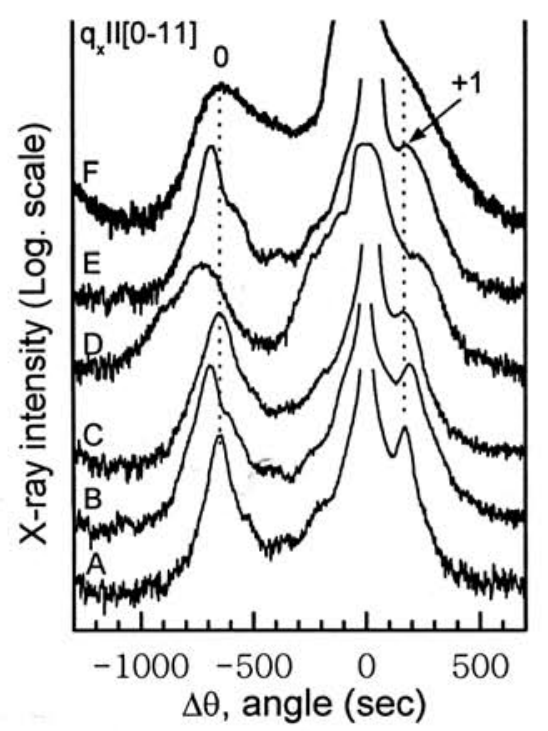

Fig. 4. Double-axis rocking curves in the range of zero and +1 satellite inherent to 17 -period $(\mathrm{In}, \mathrm{Ga}) \mathrm{As} / \mathrm{GaAs}$ quantum wires structures. The curve A correspond the initial sample. The curves B to F correspond to the RTA temperatures 550, 600, 650,750 and $850^{\circ} \mathrm{C}$, respectively. GaAs 004 reflection, $\mathrm{Cu} K_{\alpha 1}$ radiation.

annealed samples. Besides, roughness has no effect on the zero-order peak, as it is determined by the mean lattice-mismatch in the system;

3) mosaic character of the film structure, which means rotation of parts of the film area as a whole relatively to the GaAs substrate (for instance, non-uniform strains cause effective misorientation). As shown in [16], the mosaic character of the main crystal structure results in broadening that is not practically changed with the satellite order;

4) dispersion of the vertical period in such multilayer structures. Within limits close approximately to $2 \%$, this parameter does not practically influence on the width of separate satellite peaks but results in changing the relative peak intensities of the thickness oscillations [17].

As the thickness of 2D wetting layer is reduced with increasing the number of structure periods when growing the multilayer structure (in fact, it means that there exist some different periods inside this multilayer structure), roughness of interfaces as well as the composition gradients can take place. So, it should be expected an appearance of some little broadening the satellites in the initial sample as compared with the simulated $\mathrm{RC}$, which is observed in experiments (Fig. 3, curves $\mathrm{A}$ and $\mathrm{S}_{\mathrm{A}}$ ).

Thus, in our case the main contribution into broadening the reflection peaks can be caused by interface roughness, gradient of the composition and mosaic character of the film structure.
Also interesting is the fact that the angular position of reflection peaks changes with increasing the RTA temperature. The example of this angular shift observed for reflection peaks in azimuthal [0-11] direction of the radiation incidence is shown in Fig. 4. Our detailed analysis of diffraction RCs for the initial and annealed samples in the vicinity of zero satellite indicates (Fig. 4) that the increase in the RTA temperature causes an insignificant shift of this zero satellite into the side of larger angles from the GaAs peak as well as its broadening. An asymmetric character of broadening zero peak and sometimes its two-component shape (for instance, B and E curves in Fig. 4) can be caused by inhomogeneity in the composition of $2 \mathrm{D} \mathrm{InGaAs} \mathrm{layer} \mathrm{in}$ the direction of the structure growth. The angular position of this zero peak is mainly determined by the structure of 2D InGaAs layer (that is by its composition and strains) as well as by the relation thickness - strain in the layers period:

$$
\langle\varepsilon\rangle=\left(\varepsilon_{1} t_{1}+\varepsilon_{2} t_{2}\right) /\left(t_{1}+t_{2}\right),
$$

where $t_{1}, t_{2}$ and $\varepsilon_{1}, \varepsilon_{2}$ are the thickness and strain of superlattice layers period, respectively. It can be assumed that the observed shift corresponds to the increase in $x_{\mathrm{av}}$ value as a result of thermally-enhanced $\mathrm{In} / \mathrm{Ga}$ interdiffusion processes. For example, In concentration in (In, Ga)As QWRs decreases but in 2D InGaAs layer it increases. However, the latter assumption is not confirmed by the results of photoluminescence study (not shown) that indicates In concentration in (In, Ga)As QWRs to be not decreased at RTA up to $T \leq 750^{\circ} \mathrm{C}$. Another explanation of the observed structural changes can be based on the model "nucleation plus dissolution" [18]. In accord with this model, the meddle QWRs located in the lateral plane dissolves into 2D layer during the thermal annealing, which results in the increase of In concentration in 2D layer (and, possibly, in QWRs) as well as to increasing the lateral distance between QWRs. As it will be considered below, the increase in QWRs lateral period can serve as an evidence of this process actuality in our case. The effect of increasing the lateral distance between lateral columns of InAs QDs was observed after thermal annealing the multilayer structure InAs/GaAs [18]. The possible drive force of (In,Ga)As QWRs dissolution process is the presence of residual strains in the multilayer structure, which is conditioned by considerable orthogonal distortions of the structure. Therefore, when analyzing QWRs structural parameters, it is necessary to take into account not only anisotropy of quasi-periodical strains along the lateral direction [011] and growth direction [001] but possible deviations in the ordered arrangement of QWRs relatively to these two directions, which is confirmed by X-ray investigations of wire-like (In,Ga)As/GaAs quantum dot multilayers (QD chains) grown in similar technological regimes [19]. In essence, the diffusion may provide a means of relaxing strain. 
[19]. In essence, the diffusion may provide a means of relaxing strain.

Structure parameters obtained using the experimental data of HRXRD and the method of numeric modelling the experimental rocking curves.

\begin{tabular}{|c|c|c|c|c|c|c|c|c|c|}
\hline \multirow{2}{*}{$\begin{array}{l}\text { Sample } \\
T_{\text {ann }},{ }^{\circ} \mathrm{C}\end{array}$} & \multicolumn{7}{|c|}{ GaAs 004 reflection } & \multicolumn{2}{|c|}{ GaAs 311 reflection } \\
\hline & $\begin{array}{c}d_{\mathrm{GaAs}}, \\
\mathrm{nm}\end{array}$ & $\begin{array}{c}d_{\mathrm{InGaAs}}, \\
\mathrm{nm}\end{array}$ & $\begin{array}{c}\Lambda^{\perp} \\
\text { || }[011], \\
\text { nm }\end{array}$ & $\begin{array}{c}\Lambda^{\perp} \\
\mid \begin{array}{c}{[011]} \\
\mathrm{nm}\end{array} \\
\end{array}$ & $X_{\mathrm{av}}$ & $\begin{array}{c}\varepsilon_{\perp} \\
(\mathrm{GaAs})\end{array}$ & $\begin{array}{c}\varepsilon_{\perp} \\
(\mathrm{InGaAs})\end{array}$ & $\begin{array}{l}\Lambda^{\prime \prime} \\
\mathrm{nm}\end{array}$ & $\varepsilon \|(\operatorname{InGaAs})$ \\
\hline Initial & 19.7 & 3.09 & 22.79 & 22.05 & 0.27 & 0.00245 & -0.02 & 83.9 & 0.0047 \\
\hline 550 & 18.4 & 3.24 & 21.64 & 21.17 & 0.30 & 0.0025 & -0.022 & 97.8 & 0.0052 \\
\hline 600 & 19.7 & 3.27 & 22.97 & 22.93 & 0.30 & 0.0025 & -0.02 & 106.5 & 0.0056 \\
\hline 650 & 18.3 & 1.46 & 19.76 & 20.7 & 0.30 & 0.0025 & -0.022 & - & \\
\hline 750 & 18.2 & 1.3 & 19.5 & 20.36 & 0.30 & 0.0025 & -0.021 & - & \\
\hline 850 & 18.4 & 3.3 & 21.7 & 22.5 & & & & - & \\
\hline
\end{tabular}

Thus, we can assume that there is re-distribution (relaxation) of strains in the regions close to the interface QWR - 2D layer. This relaxation of residual strains, as a consequence of thermally-activated and strain-enhanced processes of $\mathrm{In} / \mathrm{Ga}$ atomic interdiffusion, is a driven mechanism of structural transformations caused by RTA of (In,Ga)As QWRs.

The samples under investigation showed good coincidence between calculated and experimental data at the level $2-5 \%$ both for the position and intensities over the whole RC. Analyzing the experimental data obtained, we determined main structural parameters of (In, Ga)As/GaAs structure.

The multilayer structure period, $\Lambda$, was determined through angular positions of any two superlattice satellites by using the relation:

$$
\Lambda=\frac{(m-n) \lambda / 2}{\sin \theta_{m}-\sin \theta_{n}}
$$

where $\lambda$ is the wavelength of $\mathrm{X}$-ray radiation $\mathrm{Cu} K_{\alpha 1}$ $(\lambda=1.54051 \AA), m(n)$ are the orders of X-ray wave $\lambda$ reflections, $\theta_{m}\left(\theta_{n}\right)$ are the reflection angles for these orders.

Stemming from the angular position of zero satellite, $\theta_{0}$, the average content of InAs, $x_{\mathrm{av}}$, in the period of the multilayer structure can be determined using the following relation:

$$
x_{\mathrm{av}}=\frac{a_{\mathrm{GaAs}}}{a_{\mathrm{InAs}}-a_{\mathrm{GaAs}}} \frac{1-v}{1+v} \cot \theta_{\mathrm{Br}}\left(\theta_{\mathrm{Br}}-\theta_{0}\right),
$$

where $a_{\mathrm{GaAs}}=5.63375 \stackrel{\circ}{\AA}\left(a_{\mathrm{InAs}}=6.0584 \stackrel{\circ}{\mathrm{A}}\right)$ is the lattice parameter of GaAs (InAs), $v=0.333$ is the Poisson coefficient, $\theta_{\mathrm{Br}}=33.02^{\circ}$ is the Bragg angle inherent to the GaAs substrate. Having determined the change of zero satellite angular position $\left(\Delta \theta_{0}\right)$ relatively to the GaAs peak from experimental reflection spectra and using the relation [20]: $\varepsilon_{\perp}=\left[\sin \theta_{\mathrm{Br}} / \sin \left(\theta_{\mathrm{Br}}-\Delta \theta_{0}\right)\right]-1$

we estimated the strain mean value $\left(\varepsilon_{\perp}\right)$ inside the layers along the direction of the structure growth. The obtained data are summarized in Table.

Note: $d_{\text {GaAs }}\left(d_{\text {InGaAs }}\right)$ is the layer thickness for GaAs (InGaAs), $\Lambda^{\perp}\left(\Lambda^{\|}\right)$is the period of the structure along the growth direction (lateral), $x_{\mathrm{av}}$ is the average In concentration determined from zero satellite position, $\varepsilon_{\perp}(\mathrm{GaAs})\left(\varepsilon_{\perp}(\mathrm{InGaAs})\right)$ is the strain along the structure growth direction for GaAs (InGaAs) layer, $\varepsilon \|(\mathrm{InGaAs})$ is the strain in the layer plane.

Shown in Fig. 5 are diffraction RCs of 004 reflection for the initial (a) and annealled at $T_{\mathrm{ann}}=750^{\circ} \mathrm{C}$ (b) samples obtained in two mutually orthogonal azimuthal directions of incident radiation: parallel (a) and perpendicular (b) relatively to orientations of (In, Ga)As QWRs. It is easy to seen that asymmetric broadening the diffraction peaks observed in the initial sample (Fig. 5a) is increased after RTA (Fig. 5b) and especially clear pronounced with growing the reflection order. As mentioned above, the main reasons for broadening these peaks can be: planar roughness of interfaces, In gradient distribution along the growth direction, mosaic character of the film structure and presence of two vertical periods in the multilayer structure. Roughness of interfaces is not a dominant effect, as we observed experimentally the change in peak angular positions [21], but this effect contribution into broadening the reflection peaks grows in the samples treated by RTA (Fig. 5b). Performed modelling the RCs with taking into account the In concentration gradient along the structure growth direction, as it was made in [22], showed that the thickness of layers containing In is too small to reach respective asymmetry of experimental reflection peaks. We suppose that the main and dominant effect providing this asymmetrical broadening the peaks is the dependence of peak angular positions on the 
a)

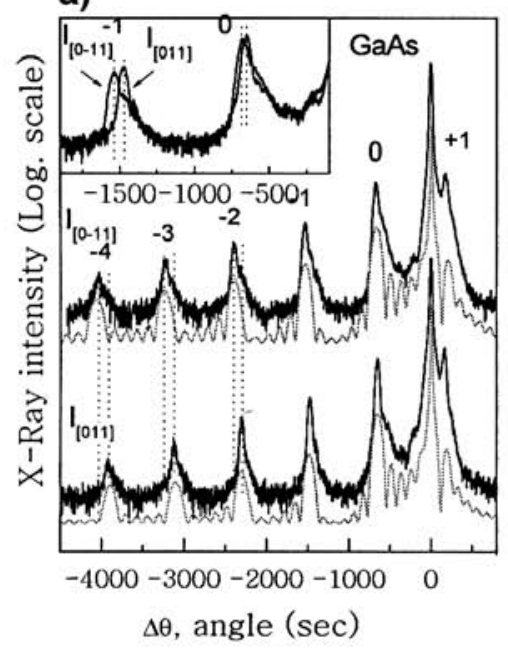

b)

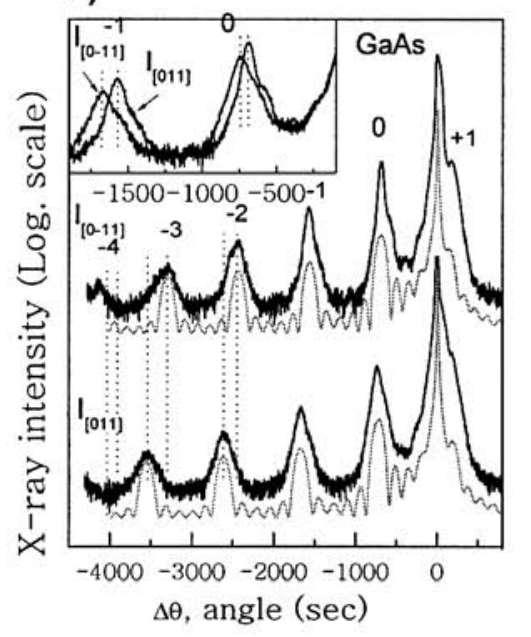

c)

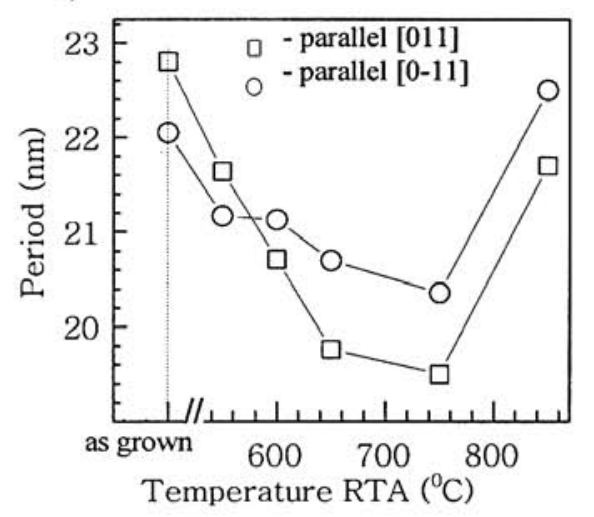

Fig. 5. Rocking curves for 004 reflection of the initial (a), annealled at $T_{\text {ann }}=750^{\circ} \mathrm{C}$ (b) samples and the dependence of the superlattice vertical period on the RTA temperature (c) for two crystallographic directions of radiation incidence: parallel $\left(q_{x} \|[0-11] \quad\left(\mathrm{I}_{[0-11]}\right)\right.$, Fig. 1a) and perpendicular $\left(q_{y} \|[011]\right.$ $\left(\mathrm{I}_{[011]}\right)$, Fig. 1b) to (In,Ga)As QWRs orientation. $\mathrm{Cu} K_{a 1}$ radiation. In the insert shown region zero and "-1" satellites. crystallographic scattering direction, which was found by us. It means that this broadening corresponds to asymmetrical splitting the reflection peaks, which can be explained by the presence of two superlattice vertical periods in the studied samples. The similar effect was observed in [23] in the course of investigations of (In, Ga)As QDs by using HRXRD method.

The sum of two interferential patterns that correspond to the structure with two vertical periods 22.05 and $22.8 \mathrm{~nm}$ in the initial sample (Fig. 5a, curves $\mathrm{S}_{\mathrm{A}}$ and $\mathrm{S}_{\mathrm{B}}$ ) is in excelent agreement with the experimental RC. Modelling the intensities of two interferential patterns was carried out using the weight ratio 10:3. The appearance of these periods is caused by a quasiperiodical elastic strain distribution that is anisotropic relatively to $\{011\}$ plane set crystallographic directions in the periodical structure (In,Ga)As/GaAs [24], this distribution being caused by the relaxation of elastic strains stimulated by quantum wires. This conclusion has been confirmed by the comparison of 004 reflection RCs with the scattering plane along [0-11] and [011] directions (Fig. 5). As to these two directions, there is a small but distinct difference of relative angular positions for zero peak and satellites of $\pm 1,-2,-3$ orders (Figs 3 to 5) relatively to the GaAs substrate peak, which is indicative of the difference of the average strain (component composition) and superlattice periods. The effect of anisotropic strain distribution is caused by anisotropy of the surface adatom diffusion. The latter results in the anisotropic distribution of built-in In atoms during formation of QWRs at the growth surface. As a consequence, there takes place the anisotropic strain distribution, which causes the orthorhombic departure from the tetragonal pseudomorphic deformation inherent to heterosystems with quantum wells.

Thus, the observed effect of splitting the satellites is a result of two effects - strain relaxation and reduction of structure symmetry. The confirmation of the assumption made is also the change in the structural vertical period value during the thermal annealing. The dependence of the period for two crystallographic directions of incident radiation on the RTA temperature is shown in Fig. 5c. It is seen that at the temperatures $T_{\mathrm{ann}} \leq 750^{\circ} \mathrm{C}$ the considerable reduction of the period is observed, while $T_{\text {ann }}=850^{\circ} \mathrm{C}$ this period increases. Besides, the period changes along [011] direction perpendicular to the QWRs orientation are significantly larger than those in [0-11] direction parallel to the QWRs orientation. It may be assumed that one of the possible reasons for changing the period is the orthorhombical distortion of 2D layer crystalline structure, which results in changing the distance , between crystallographic planes (lattice parameter) and the angle of their slope [25]. However, our estimations indicate that this reduction of the structure symmetry can change the period only by $2-3 \%$. To finally ascertain the nature of the observed changes in the period, further investigations are 

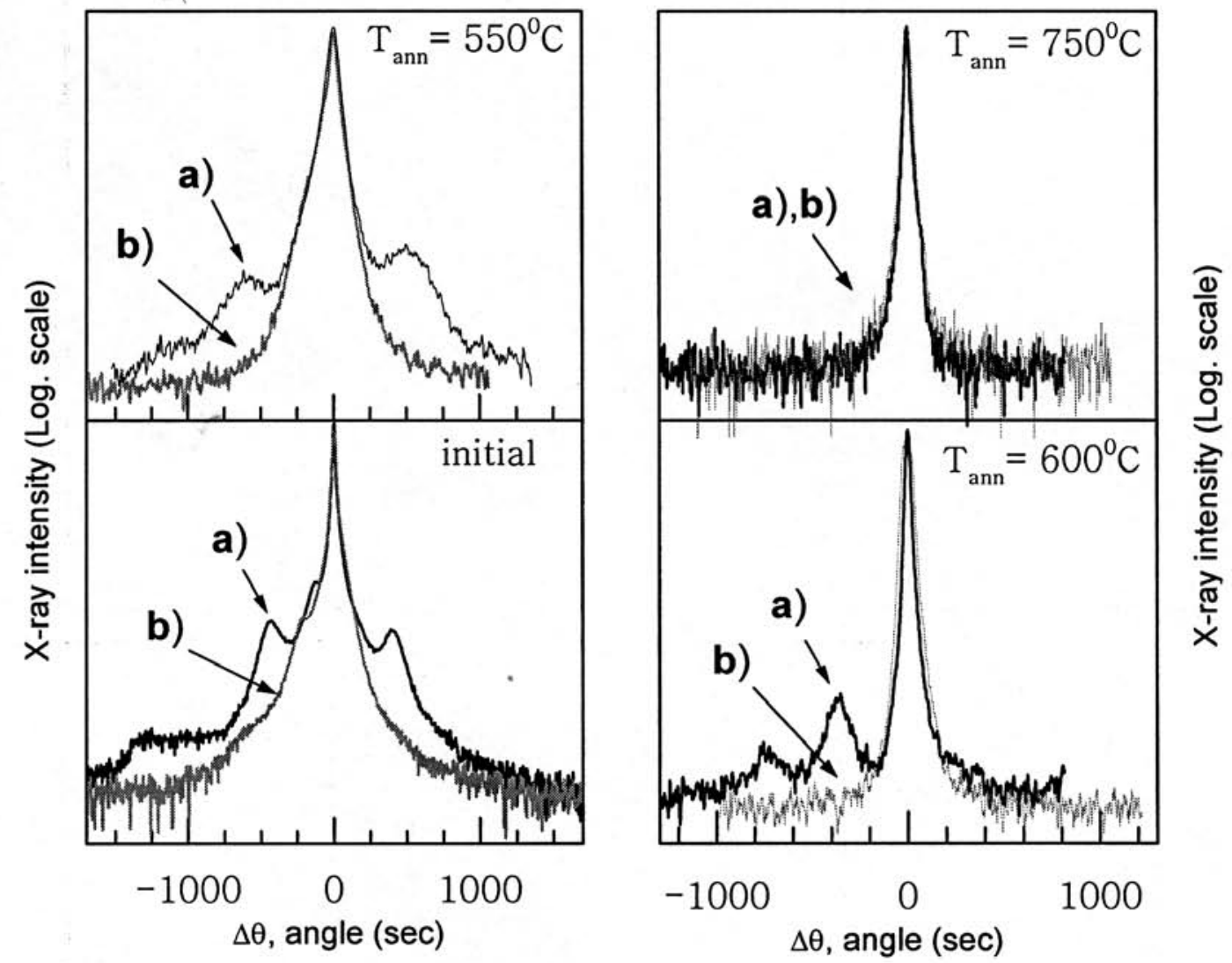

Fig. 6. X-ray diffraction spectra for the asymmetrical 311 glancing exit reflection of the 17-period (In,Ga)As/GaAs structure with (In,Ga)As QWRs (initial and annealed samples). The black (grey) spectra are measured with the incident X-ray beam parallel to the crystallographic directions: $\mathrm{a}-[011]$ and $\mathrm{b}-[0-11] . \mathrm{Cu} K_{\mathrm{a} 1}$ radiation.

necessary. At the currently stage, we can only state that the character of the structure period changes corresponds to changes in the character of the anisotropic strain distribution.

Depicted in Fig. 6 are diffraction curves for 311 reflection (in the geometry of grazing reflection beam) both for the initial and annealled samples. The experimental scheme is shown in Fig. 1c,d where the registration angle $\theta_{\mathrm{Br}-\varphi}$ is essentially smaller than the angle of incidence $\theta_{\mathrm{Br}+\varphi}$. In this case, the diffraction pattern corresponds to the multislit Fraunhofer diffraction by the surface grating [26]. In this geometry, RCs is very sensitive to lateral periodicity of modulated structures [27] as a consequence of maximizing the Xray coherent way.

RCs of the initial sample, when the beam is incident in parallel to [011] direction that is perpendicular to the direction of (In, Ga)As QWRs orientation (Fig. 6), contain the GaAs substrate central peak (i.e., zero-order peak of the diffraction grating) as well as distinct satellite peaks located symmetrically around it. There intensities are determined by QWRs geometrical sizes and internal crystalline strains within one period. RC of 311 reflection corresponds to the structure with vertically shifted and laterally ordered (In,Ga)As QWRs. When radiation is incident in parallel to [0-11] direction, that is in parallel to (In, Ga)As QW orientation, satellite peaks are not registered, which is indicative of the absence of lateral ordering in this direction.

QWRs lateral periodicity in the real space, $\Lambda$, is directly determined from the distance between satellites in the inverse space, $\Delta q_{x}$, in accord with the relation [28]:

$$
\Lambda^{\mathrm{II}}=\frac{2 \pi}{\Delta q_{x}},
$$

where $\Delta q_{x}$ is the difference between angular positions of the first satellite and that of GaAs substrate peak.

Using Eq. (5), we calculated the mean lateral period of (In,Ga)As QW for the initial sample, which is approximately equal to $83.9 \mathrm{~nm}$. It coincides well with that obtained from AFM data. As seen from Fig. 6, diffraction spectra of the annealed samples are dramatically changed. One can observe the change of satellite angular positions as well as broadening their peaks, which can testify to the lateral period change and the presence of QWRs period fluctuations (Table). At the RTA temperature $T_{\text {ann }}=750^{\circ} \mathrm{C}$, satellites are not registered, which can be caused by the loss of distinct 
QWRs lateral periodicity. The latter results in decreasing the diffracted scattering intensity to a level lower than the sensitivity of our experimental facility. Also interesting is the fact that, with increasing the RTA temperature, the lateral period grows up to $106.5 \mathrm{~nm}$ $\left(T_{\text {ann }}=600^{\circ} \mathrm{C}\right)$. It is a direct evidence of running thermally-stimulated processes of the lateral mass transfer in the form of $\mathrm{In} / \mathrm{Ga}$ atomic interdiffusion.

\section{Conclusions}

HRXRD data obtained for symmetrical and asymmetrical reflections in two mutually orthogonal directions of incident radiation (parallel and perpendicular to QWRs orientation direction) enabled to reveal anisotropy of elastic strain distribution in QWRs structure. It has been shown that, at the temperatures $T_{\text {ann }} \leq 750^{\circ} \mathrm{C}$, the driven mechanism of structural transformations is relaxation of residual strains as a consequence of thermally-activated and strain-enhanced processes of In/Ga atomic interdiffusion at the interface QWR - 2D layer, which does not result in a considerable change of In concentration in (In, Ga)As QWRs. The presence of two superlattice vertical periods and their changes after RTA in the samples under study are explained using the data about elastic strain anisotropic distribution and reduction in the structure symmetry. Also shown is that the increase in the lateral period of (In,Ga)As QWRs after RTA is caused by the processes of anisotropic strain-enhanced $\mathrm{In} / \mathrm{Ga}$ atomic interdiffusion.

\section{References}

1. K. Mukai, Y. Nakata, K. Otsubo et al., High characteristic temperature of near - 1.3- $\mu \mathrm{m}$ InGaAs/GaAs quantum-dot lasers at room temperature // Appl. Phys. Lett. 76 (23), p. 33493351 (2000); W. Huang, F. Jain, Integrated InGaAs-InP quantum wire laser-modulators for 1.55- $\mu \mathrm{m}$ applications // Opt. Eng. 43 (3), p. 667672 (2004).

2. M.Ya. Valakh, V.V. Strelchuk, A.F. Kolomys et al., Resonant Raman scattering and atomic force microscopy of InGaAs/GaAs multilayer nanostructures with quantum dots // Semiconductors 39(1), p. 127-131 (2005).

3. V.V. Strelchuk, M.Ya. Valakh, A.F. Kolomys, P.M. Lytvyn et al., Vertical and lateral ordering the quantum dots and quantum wires in $\mathrm{In}_{\mathrm{x}} \mathrm{Ga}_{1-\mathrm{x}} \mathrm{As} / \mathrm{GaAs}(100)$ multilayer structures // Abstracts of $2^{\text {nd }}$ Ukrainian scientific conference for physics of semiconductors, 20-24 September, 2004, Vol. 2, p. 220-221.

4. W. Ma, R. Nötzel, A. Trampert et al., Selforganized quantum wires formed by elongated dislocation-free islands in (In,Ga)As/GaAs(100) // Appl. Phys. Lett. 78 (9), p. 1297-1299 (2001).

5. R. Leon, Y. Kim, C. Jagadish, M. Gal, J. Zou, D.J.H. Cockayne, Effects of interdiffusion on the luminescence of InGaAs/GaAs quantum dots // Appl. Phys. Lett. 69(13), p.1888-1890 (1996).

6. S.J. Xu, X.C. Wang, S.J. Chua, C.H. Wang et al., Effects of rapid thermal annealing on structure and luminescence of self-assembled InAs/GaAs quantum dots // Appl. Phys. Lett. 72 (25), p. 33353337(1998); S.J. Xu, H. Wang, Q. Li et al., X-ray diffraction and optical characterization of interdiffusion in self-assembled InAs/GaAs quantum-dot superlattices // Ibid. 77(14), p. 21302132 (2000).

7. J. Tersoff, C. Teichert, M.G. Lagally, Selforganization in growth of quantum dot superlattices // Phys. Rev. Lett. 76 (10), p. 1675 1678 (1996).

8. N.N. Faleev, Yu.G. Musikhin, A.A. Suvorov, A.Yu. Yegorov, A.Ye. Zhukov, A.R. Kovsh, V.M. Ustinov, M. Tabuchi, Y. Takeda, X-ray and synchrotron diffraction and transmission electron microscopy studies of anisotropy of spatial distribution of $\operatorname{In}(\mathrm{Ga})$ As quantum dots in In(Ga)As/GaAs multilayer heterostructures // Fiz. Tekhnika Poluprov. 35(8), p. 969-978 (2001) (in Russian).

9. L. Tapfer, P. Sciacovelli, L. De Caro, Double- and triple-crystal X-ray diffraction analysis of semiconductor quantum wires // J. Phys. D: Appl. Phys. 28, p. A179-A173(1995).

10. A. Ponchet, A. Rocher, A. Ougazzaden, A. Mircea, Self-induced laterally modulated GaInP/InAsP structure grown by metal-organic vapor-phase epitaxy // J. Appl. Phys. 75 (12), p. 7881-7883 (1994).

11. R.L. Headrick, J.-M. Baribeau, Y.E. Strausser, Anisotropic roughness in $\mathrm{Ge} / \mathrm{Si}$ superlattices // Appl. Phys. Lett. 66 (1), p. 96-98 (1995).

12. Z.M. Wang, K. Holms, Yu.I. Mazur, G.J. Salamo, Fabrication of (In, Ga)/As quantum-dot chains on GaAs(100) // Ibid. 84 (11), p. 1931-1933 (2004).

13. A. Krost, F. Heinrichsdor, D. Bimberg, A. Darhuber, G. Bauer, High-resolution X-ray diffraction of self-organized InGaAs/GaAs quantum dot structures // Ibid. 68 (6), p. $785-787$ (1996).

14. D. Pal, E. Towe, S. Chen, Structural characterization of InAs/GaAs quantum-dot nanostructures // Ibid. 78 (26), p. 4133-4135 (2001).

15. Yu.I. Mazur, W.Q. Ma, X. Wang et al., InGaAs/GaAs three-dimensionally-ordered array of quantum dots // Ibid. 83 (5), p. 987-989 (2003).

16. V. Holy, J. Kubena, E. Abramof, A. Presek, E. Koppensteiner // X-ray diffractometry of small defects in layered systems // J. Phys. D: Appl. Phys. 26 (4A), p. A146-A150 (1993). 
17. A.K. Bowen, B.K. Tanner, High resolution X-ray diffractometry and topography. St.-Petersburg, Nauka (2002).

18. W. Chen, B. Shin, R.S. Goldman, A. Stiff, P.K. Bhattacharya, Mechanisms of lateral ordering of InAs/GaAs quantum dot superlattices // J. Vac. Sci. Technol. B 21 (4), p. 1920-1923 (2003).

19. D. Grigoriev, M. Schmidbauer, P. Schaefer et al., Three-dimensional self-ordering in an InGaAs/GaAs multilayer quantum dot structure investigated by X-ray diffuse scattering // J. Phys. D: Appl. Phys. (2004) (in press).

20. S. Krishna, S. Raghavan, A.L. Gray, A. Stintz, K.J. Malloy, Characterization of rapid-thermalannealed InAs/ $/ \mathrm{In}_{0.15} \mathrm{Ga}_{0.85} \mathrm{As}$ dots-in-well heterostructure using double crystal X-ray diffraction and photoluminescence // Appl. Phys. Lett. 80 (21), p. 3898-3900 (2002).

21. I.K. Schuller, M. Grimsditch, F. Chambers et al. // Phys. Rev. Lett. 65 (10), p. 1235-1238 (1990).

22. A. Krost, G. Bauer, J. Woitok, High resolution $X$-ray diffraction in: optical characterization of epitaxial semiconductor layers, Eds G. Bauer, W. Richter; Springer, Berlin (1996) p. 347.

23. A. Krost, F. Heinrichsdorff, D. Bimberg, J. Bläsing, A. Darhuber, G. Bauer, X-ray analysis of self-organized InAs/InGaAs quantum dot structures // Cryst. Res. Technol. 34(1) p.89-102 (1999).
24. N. Faleev, K. Pavlov, M. Tabuchi, and Y. Takeda, Influence of long-range lateral ordering in structures with quantum dots on the special distribution of diffracted X-ray radiation // Jpn J. Appl. Phys. 38, Part 1(2A), p.818-821 (1999).

25. A. Sanz-Hervas, M. Aguilar, J.L. Sanchez-Rojas et $a l$, Observation of non-trigonal lattice distortion in pseudomorphic InGaAs/GaAs superlattices grown on misoriented (111)B GaAs // J. Appl. Phys. 82(7), p. 3297-3305 (1997).

26. L. De Caro, P. Sciacovelli, and L. Tapfer, Doublecrystal X-ray diffraction from periodically corrugated crystalline semiconductor surfaces // Appl. Phys. Lett. 64, (1), p.34-36 (1994).

27. W. Ma, R. Nötzel, A. Trampert et al., Selforganized quantum wires formed by elongated dislocation-free islands in (In,Ga)As/GaAs(100) // Appl. Phys. Lett. 78 (9), p.1297-1299 (2001); T. Mano, R. Nötzel, G.J. Hamhuis et al., Effect of annealing on formation of self-assembled (In,Ga)As quantum wires on GaAs (100) by molecular beam epitaxy // J. Appl. Phys. 92 (7), p. 4043-4046 (2002).

28. Qun Shen, Stefan W. Kycia, E.S. Tentarelli, W.J. Schaff, and L.F. Eastman, X-ray-diffraction study of size-dependent strain in quantum-wire structures. // Phys. Rev. B 54 (23), p. 16381-16384 (1996). 